Glória de Paula Silva (Orcid: 0000-0002-6344-4237) ${ }^{1}$ Ravini de Souza Sodré (Orcid: 0000-0003-0413-0497) Daianny França Pinto (Orcid: 0000-0003-3158-4283) ${ }^{1}$ Priscila dos Santos Bunn (Orcid: 0000-0001-6193-4788) Elirez Bezerra da Silva (Orcid: 0000-000-5989-6556)

\section{ASSOCIAÇÃO DA MOBILIDADE TORACOABDOMINAL NAS POSIÇÕES ORTOSTÁTICA E DECÚBITO DORSAL COM AS PRESSÕES INSPIRATÓRIA E EXPIRATÓRIA MÁXIMAS (PIMÁX E PEMÁX)}

\author{
ASSOCIATION OF THORACOABDOMINAL MOBILITY \\ IN THE ORTOSTATIC AND SUPINE POSITIONS WITH \\ THE MAXIMUM INSPIRATORY AND EXPIRATORY \\ PRESSURES (MIP AND MEP)
}

\begin{abstract}
RESUMO
Objetivos: verificar se existe diferença na mobilidade toracoabdominal (MTA) entre as posições ortostática e decúbito dorsal e correlacionar a MTA com valores de pressões inspiratória e expiratória máximas (PImáx e PEmáx), em indivíduos saudáveis de ampla faixa etária e de ambos os sexos. Método: foram avaliados 276 participantes, sendo 130 homens e 146 mulheres, com idade de 47,03 $\pm 17,10$ anos. A MTA foi familiarizada e avaliada nas posições ortostática e decúbito dorsal, com a utilização de uma trena antropométrica nos níveis axilar, xifoide e umbilical durante três manobras inspiratórias e expiratórias máximas. As PImáx e PEmáx foram familiarizadas e avaliadas conforme os procedimentos da Sociedade Brasileira de Pneumologia e Tisiologia. A normalidade dos dados foi verificada pelo teste de Kolmogorov-Smirnov. O teste t pareado foi adotado para comparar as medidas da MTA nas posições ortostática e decúbito dorsal; e a Correlação de Pearson (r), para avaliar a associação da MTA com as PImáx e PEmáx. Resultados: O teste t pareado mostrou diferenças significativas da MTA nos níveis axilar, xifoide e umbilical, quando mensurada nas posições ortostática e decúbito dorsal. A MTA diminuiu significativamente de 2 $\mathrm{mm}(3 \%)$ ao nível axilar e $7 \mathrm{~mm}$ (13\%) ao nível xifoide na posição decúbito dorsal. Já na posição ortostática, a MTA diminuiu de $18 \mathrm{~mm}$ (28\%) ao nível umbilical em relação ao decúbito dorsal. A MTA ao nível umbilical teve uma diminuição significativa de $18 \mathrm{~mm}(28 \%)$ na posição ortostática $(4,6 \pm 2,1)$ comparada à posição decúbito dorsal $(6,4 \pm 2,0)$. Conclusão: comparando-se à posição ortostática, o decúbito dorsal promove diminuição da MTA ao nível axilar e xifoide e um aumento significativo ao nível umbilical. Entretanto, a correlação da MTA com as PImáx e PEmáx é, respectivamente, moderada e fraca.
\end{abstract}

Palavras-chave: Tórax; Mobilidade toracoabdominal; Posição ortostática; decúbito dorsal; Cirtometria.

\begin{abstract}
Objectives: to verify if there is a difference in the MTA between the orthostatic and supine positions, and to correlate the MTA with values of maximum inspiratory and expiratory pressures(MIP and MEP), in healthy individuals of a wide age group and of both sexes. Method: 276 participants were evaluated, 130 men and 146 women, aged 47.03 \pm 17.10 years. The MTA was familiarized and evaluated in the orthostatic and supine positions, using an anthropometric measuring tape at the axillary, xiphoid and umbilical levels during three maximum inspiratory and expiratory maneuvers. MIP and MEP were familiarized and evaluated according to the procedures of the Brazilian Society of Pulmonology and Tisiology. The normality of the data was verified by the Kolmogorov-Smirnov test. The paired t-test was adopted to compare MTA measurements in the orthostatic and supine positions and Pearson's Correlation (r) to assess the association of thoracoabdominal mobility with MIP and MEP. Results: the normality of the data was verified by the Kolmogorov-Smirnov test. The paired t-test showed significant differences in MTA at the axillary, xiphoid and umbilical levels, when measured in the orthostatic and supine positions. The MTA decreased significantly from $2 \mathrm{~mm}$ (3\%) at axillary levels and $7 \mathrm{~mm}(13 \%)$ at xiphoid level in the supine position. In the orthostatic position, the MTA decreases from $18 \mathrm{~mm}(28 \%)$ to the umbilical level in relation to the supine position. The MTA at the umbilical level had a significant decrease of $18 \mathrm{~mm}(28 \%)$ in the orthostatic position $(4.6 \pm 2.1)$ compared to the supine position $(6.4 \pm 2.0)$. MTA showed medium and low correlation with MIPs, and medium and low medium with MIPs, and had a greater relationship with the supine position. Conclusion: it is suggested the adoption of the supine position for the evaluation of the MTA for the reasons already presented. Comparing to the orthostatic position, the supine position promotes a decrease in the MTA at the axillary and xiphoid level and a significant increase at the umbilical level. However, the correlation of MTA with MIP and MEP is moderate and weak, respectively.
\end{abstract}




\section{INTRODUÇÃO}

As alterações dos volumes pulmonares estão intrinsecamente associadas à movimentação do gradil costal durante as fases respiratórias, que, em conjunto com os músculos ventilatórios, viabilizam de maneira coordenada a entrada e a saída de gases dos pulmões ${ }^{1,2}$. A avaliação tanto da expansibilidade torácica quanto da musculatura ventilatória tem sido importante indicador clínico de doenças que acometem o sistema respiratório ${ }^{3}$ e utilizada no acompanhamento de programas de reabilitação $0^{4,5}$.

A amplitude do tórax pode ser mensurada pela mobilidade toracoabdominal (MTA), que consiste em avaliar os movimentos da caixa torácica e do abdômen durante manobras inspiratórias e expiratórias máximas ${ }^{6}$, com a utilização de uma trena antropométrica posicionada nos níveis axilar, xifoide e umbilical ${ }^{2}$. Já a força dos músculos ventilatórios é verificada pelas pressões inspiratória e expiratória máximas (PImáx e PEmáx), de maneira indireta, com o instrumento manovacuômetro ${ }^{7,8}$.

Embora as posições ortostática e decúbito dorsal sejam comumente utilizadas para a avaliação da MTA, não há nenhuma padronização quanto à postura a ser adotada, apesar do sistema respiratório sofrer modificações conforme o posicionamento corporal. $\mathrm{Na}$ postura ortostática, há uma vantagem maior na mecânica ventilatória ao nível torácico; e na posição decúbito dorsal, a ação gravitacional favorece a respiração abdominal ${ }^{9}$. Há, portanto, uma complexa interação entre as posturas corporais, os movimentos respiratórios e a MTA que devem ser mais estudadas para nortear os profissionais nas tomadas de decisões quanto a sua avaliação.

Considerando isso, os objetivos deste estudo foi verificar se existe diferença na MTA entre as posições ortostática e decúbito dorsal e correlacionar a MTA com valores de PImáx e PEmáx, em indivíduos saudáveis de ampla faixa etária e de ambos os sexos.

\section{MÉTODO}

Este estudo, tipo transversal, foi redigido a partir das recomendações do Strengthening the Reporting of Observational Studies in Epidemiology $(\text { Strobe })^{10}$.

\section{Delineamento}

O estudo foi realizado em centros esportivos, localizados na Baixada Fluminense, na Zona Oeste e na Zona Norte do estado do Rio de Janeiro.

Investigou-se um único grupo em três momentos distintos: avaliação da MTA na posição decúbito dorsal versus ortostática e avaliação das PImáx e PEmáx. Os participantes foram convidados para a pesquisa de forma voluntária e receberam informação referente ao local de realização do estudo e processo de coleta de dados, que foram esclarecidos pessoalmente ou por contato telefônico. Ao confirmarem a participação, realizou-se uma triagem; e após serem elegíveis, foram avaliadas a MTA e as PImáx e PEmáx. Tanto a triagem quanto as avaliações foram conduzidas por profissionais de Educação Física.

As coletas de dados foram realizadas cinco vezes por semana (de segunda a sexta), das 7 horas às 20 horas; $e$ as medidas da MTA nas posições ortostática e decúbito dorsal, bem como as medidas de PImax e PEmax, foram verificadas no mesmo dia.

\section{Amostra}

A amostra foi não probabilística, composta por 276 participantes, sendo 130 homens e 146 mulheres, na faixa etária compreendida entre 20 e 79 anos. Os critérios de inclusão adotados foram: participantes saudáveis, com índice de massa corporal (IMC) igual ou superior a $(18,5 \mathrm{~kg} / \mathrm{m} 2)$ e igual ou inferior a $(29,9$ $\mathrm{kg} / \mathrm{m} 2)^{11}$, sem histórico passado ou presente de tabagismo, sem deformidades torácicas como pectus carinatum ou 
excavatum, não portadores de doenças cardiorrespiratórias ou neuromusculares. Os participantes foram notificados sobre os procedimentos do estudo e assinaram o Termo de Consentimento Livre e Esclarecido concordando em participar da pesquisa, de acordo com a Resolução 466, de 12 de dezembro de 2012 , do Conselho Nacional de Saúde. A presente pesquisa foi aprovada pelo Comitê de Ética em Pesquisa da Universidade do Estado do Rio de Janeiro (CEP/Uerj), sob o Parecer de n ${ }^{\circ} 1.799 .360$.

\section{Procedimentos}

O estudo foi organizado da seguinte forma: 1) agendamento e informações das condições prévias para avaliação da MTA e das PImáx e PEmáx , que compreenderam a seguinte recomendação: não consumir refeição completa nas três horas antecedentes; 2) triagem dos participantes; 3 ) avaliação do nível de atividade física; 4) familiarização e avaliação da MTA e das PImáx e PEmáx. As etapas $1), 2$ ) e 3 ) foram realizadas por duas pesquisadoras, e a etapa 4) foi realizada pela pesquisadora responsável.

\section{Triagem dos participantes}

$\mathrm{Na}$ triagem, foi realizada anamnese dos participantes para elegibilidade ao estudo com perguntas referentes aos critérios de inclusão; verificação da massa corporal total e da estatura para avaliação do IMC; dados sociodemográficos, como idade, prática de exercícios físicos (tipos de exercícios, regularidade e frequência).

\section{Avaliação da MCT e estatura}

A estatura foi verificada com a utilização de um estadiômetro portátil/ Sanny com capacidade máxima para 2,10 m e acurácia de $0,001 \mathrm{~m}$. A MCT foi aferida com uma balança digital G-tech limitada em $150 \mathrm{Kg}$ e precisão de $0,1 \mathrm{Kg}$. Em ambas as medições antropométricas, foram adotados os específicos protocolos propostos pela International Standards for Anthropometric Assessment ${ }^{12}$. Com base nas referidas medidas, foi calculado o IMC.

$\frac{3}{\text { Silva et al }}$

Avaliação do nível de atividade física

O nível de atividade física foi avaliado pelo Questionário Internacional de Atividade Física (Ipaq) ${ }^{13}$, em sua versão curta e validada para a população brasileira.

Familiarização e avaliação da MTA

A MTA foi verificada por meio da cirtometria $^{2,14}$, com a utilização de uma trena antropométrica Sanny, de $2 \mathrm{~m}$ de comprimento com escala em milímetros, e com o participante nas posições ortostática e decúbito dorsal, com os membros superiores estendidos ao lado do corpo e com o tórax descoberto. Foram medidas as circunferências em três níveis na seguinte ordem: axilar, xifoide e umbilical, após uma inspiração máxima até a capacidade pulmonar total; e após uma expiração máxima até o volume residual.

O nível axilar teve como ponto de referência o manúbrio (parte do osso esterno), que foi demarcado a partir de uma medida palpatória com os dedos indicador e médio posicionados horizontalmente abaixo da incisura jugular (parte superior do manúbrio); o nível xifoide foi localizado no processo xifoide, na parte inferior do esterno; e o nível umbilical foi demarcado em cima da cicatriz umbilical ${ }^{15}$.

A familiarização foi realizada para determinar e marcar os níveis anatômicos com uma caneta esferográfica na superfície corporal dos voluntários e ambientar os participantes com as manobras inspiratórias e expiratórias máximas. Em seguida, cada nível foi contornado com a trena antropométrica com seu valor zero fixado sobre o ponto de referência. Cada nível anatômico foi mensurado três vezes durante as manobras inspiratórias e expiratórias, com intervalos de um minuto intra e interposições.

$\mathrm{Na}$ avaliação, cada nível foi verificado três vezes durante os movimentos inspiratórios e expiratórios, com intervalos de 
um minuto intra e interposições. Registraram-se o maior valor inspiratório e o menor valor expiratório de cada nível (axilar, xifoide e umbilical). A MTA de cada nível foi calculada pela diferença entre esses valores ${ }^{5,15}$.

O tempo de intervalo entre a familiarização e a avaliação foi de, no mínimo, 3 minutos.

Familiarização e avaliação das PImáx e PEmáx

A familiarização e a avaliação das PImáx e PEmáx foram realizadas conforme os procedimentos propostos pelas diretrizes para testes de função pulmonar ${ }^{1,7,11}$, com o participante sentado em uma cadeira com o encosto formando um ângulo de aproximadamente $90^{\circ}$ em relação ao quadril, com os membros superiores estendidos ao longo do tronco, os joelhos fletidos em torno de $90^{\circ}$ e os pés apoiados no solo ${ }^{1,6,11,15}$. Em seguida, o participante foi orientado a manter os lábios firmes no bocal e utilizar um clipe nasal, para evitar o escapamento de ar e a perda de pressão durante a medição ${ }^{1,6,11,13,15}$.

Posteriormente, a musculatura facial dos participantes foi comprimida, a fim de evitar que a contração dos músculos bucinadores aumentasse a pressão intraoral e interferisse no resultado da medição ${ }^{8}$. Logo, os participantes foram orientados a realizar uma inspiração máxima, próxima à capacidade pulmonar total, e uma expiração máxima, próxima ao volume residual por meio de um bocal, sendo motivados para que o teste fosse executado corretamente. Para a familiarização, foram realizadas cinco medidas para PImáx e PEmáx, com o intervalo de 1 minuto intramedições e de 3 minutos interpressões ${ }^{16,17}$. Para as avaliações das PImáx e PEmáx, realizou-se um mínimo de três e máximo de cinco medidas, com intervalo de 1 minuto intramedições e de 5 minutos interpressões ${ }^{16,17}$.

Foram consideradas como reprodutíveis medidas de PImáx e PEmáx igual ou não superior a $10 \%$ entre três e cinco tentativas máximas ${ }^{1,6,11,13,15}$. As
PImáx e PEmáx foram mensuradas por um manovacuômetro digital $\mathrm{MDV}^{\oplus} 300$ (MDI/BRASIL) com resolução de 1 cmH2O, intervalo operacional de \pm 300 cmH2O, com certificado de calibração emitido pelo Inmetro sob o número 0467/2015. Para conectar o bocal de plástico tipo voldyne com orifício de fuga de $2 \mathrm{~mm}$ ao manovacuômetro, foram utilizados um conector rescal plástico padrão; um isolador de contaminação bacteriana e umidade no transdutor de pressão; um tubo liso transparente de silicone com $37 \mathrm{~cm}$ de comprimento e $5 \mathrm{~mm}$ de diâmetro externo. As avaliações das PImáx e PEmáx foram realizadas pela pesquisadora responsável.

Os compartimentos do instrumento foram esterilizados com uma solução antimicrobiana à base de hipoclorito de sódio.

Análise dos dados

A normalidade dos dados foi verificada pelo teste de Kolmogorov-Smirnov. Atendidos os pressupostos, realizou-se uma análise descritiva com frequências absolutas e relativas. $\mathrm{O}$ teste $\mathrm{t}$ pareado foi adotado para comparar a MTA nas posições ortostática e decúbito dorsal. A correlação de Pearson foi utilizada para verificar a associação entre a MTA e as PImáx e PEmáx. A magnitude da correlação foi classificada de acordo com os seguintes limiares: $r=0,10$ 0,29 (pequena), 0,30-0,49 (moderada), 0,50-0,69 (grande), 0,70-0,89 (muito grande), 0,90-0,99 quase perfeita ou 1,00 (perfeita) $)^{18}$

O nível de significância estabelecido para todos os testes foi de $\mathrm{P} \leq 0,05$. Todas as análises estatísticas foram realizadas por meio do programa Statistica 7, Stat Soft, Inc. 1984-2004.

\section{RESULTADOS}

Foram avaliados 276 participantes, sendo 130 homens e 146 mulheres. Os 
níveis de atividade avaliados pelo Ipaq ${ }^{13}$ dos participantes em frequência absoluta e relativa foram: muito ativo $(24 / 8,6)$; ativo (125 / 45,0); irregularmente ativo A (77 / 17,7); irregularmente ativo B (45 / 16,2) e sedentário $(7 / 2,5)$.

As características antropométricas, MTA, PImax e PEmax dos participantes estão apresentadas na Tabela 1.

Tabela 1. Análise descritiva das características da amostra, da pressão inspiratória máxima, da pressão expiratória máxima e da mobilidade toracoabdominal $(\mathrm{n}=276)$.

\begin{tabular}{|c|c|c|c|}
\hline Variáveis & Média士DP & Mínimo & Máximo \\
\hline \multicolumn{4}{|l|}{ Características da amostra } \\
\hline Idade (anos) & $47,03 \pm 17,10$ & 20,00 & 79,00 \\
\hline Massa corporal total $(\mathrm{Kg})$ & $69,54 \pm 11,00$ & 46,70 & 94,40 \\
\hline Estatura (m) & $1,66 \pm 0,09$ & 1,41 & 1,91 \\
\hline \multicolumn{4}{|l|}{ MTA Ortostática } \\
\hline Axilar $(\mathrm{cm})$ & $6,04 \pm 1,77$ & 1,80 & 14,80 \\
\hline Xifoide (cm) & $5,99 \pm 1,89$ & 1,40 & 14,80 \\
\hline Umbilical (cm) & $4,65 \pm 2,07$ & 0,20 & 13,80 \\
\hline \multicolumn{4}{|l|}{ MTA Decúbito dorsal } \\
\hline Axilar (cm) & $5,78 \pm 1,83$ & 1,40 & 10,90 \\
\hline Xifoide $(\mathrm{cm})$ & $5,35 \pm 2,25$ & 0,70 & 13,80 \\
\hline Umbilical (cm) & $6,37 \pm 1,97$ & 1,50 & 14,20 \\
\hline PImáx $\left(\mathrm{cmH}_{2} \mathrm{O}\right)$ & $107,49 \pm 34,84$ & 24 & 191 \\
\hline PEmáx $\left(\mathrm{cmH}_{2} \mathrm{O}\right)$ & $146,27 \pm 29,24$ & 45 & 247 \\
\hline
\end{tabular}

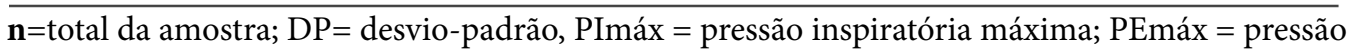
expiratória máxima; MTA = mobilidade toracoabdominal. 
significativas na MTA quando mensuradas nas posições ortostática e decúbito dorsal (Figuras 1, 2 e 3).

Figura 1. Mobilidade toracoabdominal ao nível axilar nas posições ortostática e decúbito dorsal $(\mathrm{cm})$

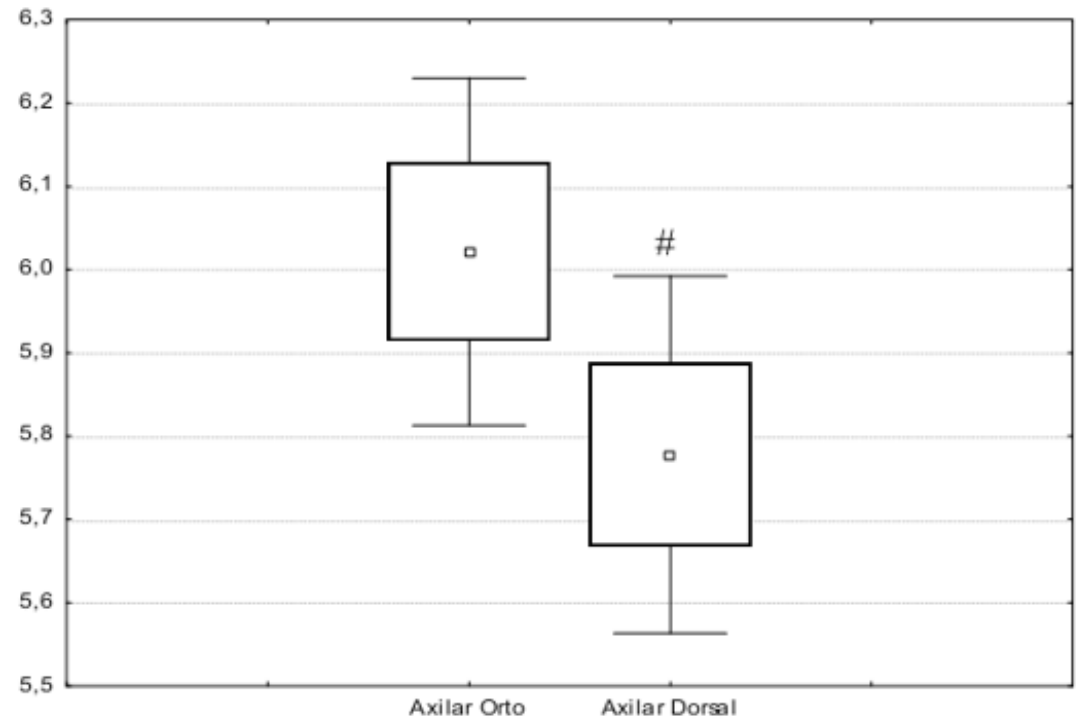

(Quadrado menor = média; Quadrado maior = média \pm erro padrão; hastes = média $\pm 1,96$ erro padrão; \# $\mathrm{t}=2,6 ; \mathrm{p}=0,01)$

Figura 2. Mobilidade toracoabdominal ao nível xifoide nas posições ortostática e decúbito dorsal

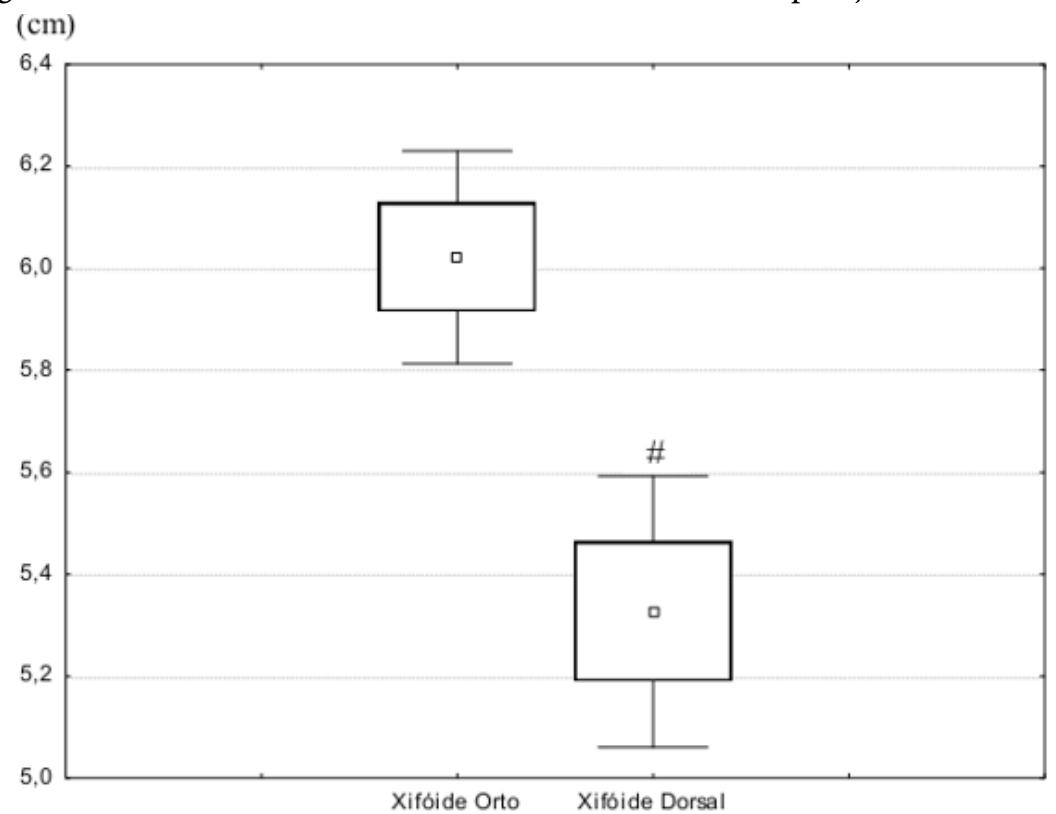

(Quadrado menor $=$ média; Quadrado maior $=$ média \pm erro padrão; hastes $=$ média $\pm 1,96$ erro padrão; $\# \mathrm{t}=5,6 ; \mathrm{p}=0,0000001)$ 
Figura 3. Mobilidade toracoabdominal ao nível umbilical nas posições ortostática e decúbito dorsal

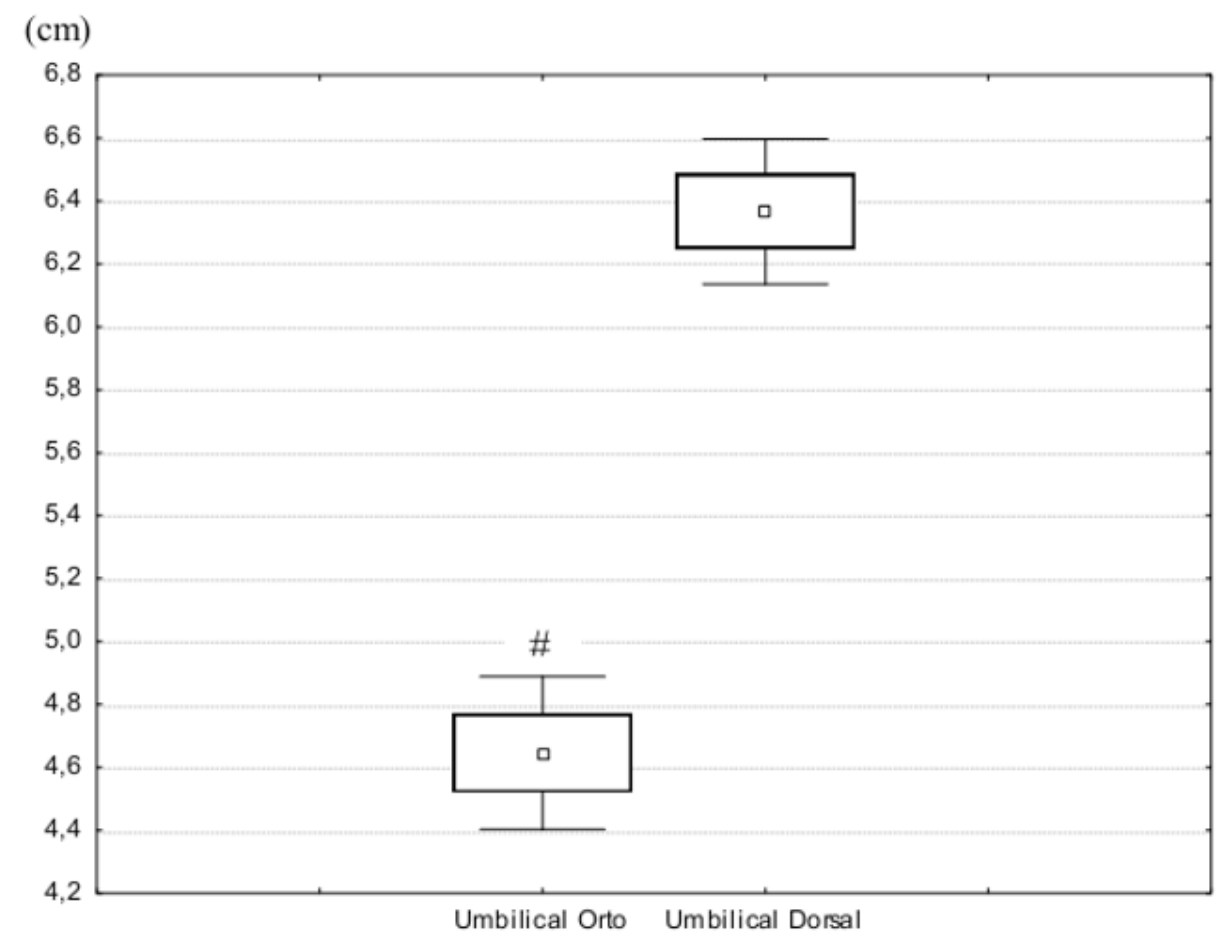

(Quadrado menor $=$ média; Quadrado maior $=$ média \pm erro padrão; hastes $=$ média $\pm 1,96$ erro padrão; $\# \mathrm{t}=-14,3 ; \mathrm{p}=0,001$ )

A correlação da MTA com as PImax

e PEmax estão apresentadas na Tabela 2.

Tabela 2. Correlação entre a mobilidade toracoabdominal e a pressão inspiratória e expiratória máximas (PImáx e PEmáx)

\begin{tabular}{|c|c|c|c|c|c|c|}
\hline \multirow{2}{*}{ Variáveis } & \multicolumn{3}{|c|}{ Postura ortostática $(\mathrm{cm})$} & \multicolumn{3}{|c|}{ Postura dorsal (cm) } \\
\hline & Axilar & Xifoide & Umb. & Axilar & Xifoide & Umb. \\
\hline PImáx $\left(\mathrm{cmH}_{2} \mathrm{O}\right)$ & $0,36^{*}$ & $0,36^{*}$ & $-0,06$ & $0,38^{*}$ & $0,31^{*}$ & $0,19 *$ \\
\hline PEmáx $\left(\mathrm{cmH}_{2} \mathrm{O}\right)$ & $0,25^{*}$ & $0,25^{*}$ & 0,04 & $0,31^{*}$ & $0,33^{*}$ & $0,22^{*}$ \\
\hline
\end{tabular}

Umb. = umbilical; PImáx $=$ pressão inspiratória máxima; PEmáx $=$ pressão expiratória máxima. ${ }^{*} \mathrm{P}<0,05$ 


\section{DISCUSSÃO}

A avaliação da MTA é um conjunto de medidas aos níveis axilar, xifoide e umbilical, que permite realizar o exame físico do tórax pelos movimentos torácicos e abdominais de maneira simples e baixo custo, podendo ser realizada em qualquer ambiente com a utilização apenas de uma trena antropométrica ${ }^{2,19}$. Apesar de a literatura não apontar um padrão sobre as posturas a serem adotadas, alguns estudos avaliaram a MTA com participantes de distintas faixas etárias em ortostatismo ${ }^{5,20-22} \mathrm{e}$ em decúbito dorsal ${ }^{3,6,23}$.

Os resultados do presente estudo mostraram diferenças significativas na MTA nos níveis axilar, xifoide e umbilical. Após passar da posição ortostática para decúbito dorsal, a MTA diminuiu significativamente de $2 \mathrm{~mm}$ (3\%) ao nível axilar, de $7 \mathrm{~mm}(13 \%)$ ao nível xifoide, porém aumentou $18 \mathrm{~mm}$ (28\%) ao nível umbilical (Tabela 1 e Figuras 1 , 2 e 3). O aumento da MTA aos níveis axilar e xifoide na posição ortostática pode ter ocorrido devido ao trabalho dos músculos ventilatórios ao nível costal sem a ação da gravidade e da contrarresistência do chão ou maca, enquanto o aumento da MTA ao nível umbilical pode ter sido pelo deslocamento do diafragma, relacionado com o seu comprimento, sendo menos eficiente na posição ortostática ${ }^{25}$. Esses resultados são corroborados pelo estudo de Pedrini et al. ${ }^{14}$, que comparou as medidas da MTA nas posições ortostática e decúbito dorsal, em 29 participantes saudáveis na faixa etária de 27,8 $\pm 4,4$ anos, encontrando aumento significativo $(\mathrm{P}<0,001)$ da MTA ao nível umbilical de 2,79 $\pm 1,79 \mathrm{~cm}$ em ortostática para $4,25 \pm 2,08 \mathrm{~cm}$ em decúbito dorsal. O presente estudo mostrou diferenças significativas nos três níveis avaliados (axilar, xifoide e umbilical), com aumento de $52 \%$ ao nível umbilical ao passar da postura ortostática para a decúbito dorsal. Pedrini et al. ${ }^{14}$ podem não ter encontrado diferenças significativas aos níveis axilar e xifoide, uma vez que as magnitudes nesses níveis foram menores e requereriam uma amostra maior. Outros estudos que utilizaram magnetômetros ou pletismografia ${ }^{25,26}$ encontraram também maior mobilidade abdominal na postura decúbito dorsal. Uma possível explicação pode ser uma melhor relação de comprimento-tensão do diafragma em decorrência do deslocamento superior das vísceras abdominais ${ }^{14}$.

A associação da MTA com as PImax e PEmax na posição ortostática, apesar de significativas, exceto para o nível umbilical, não ultrapassou a classificação de moderada. Na posição decúbito dorsal, foi significativa nos três níveis, porém moderada também ${ }^{18}$ (Tabela 2). Esses resultados foram corroborados pelo estudo de Lanza et al. ${ }^{16}$, que encontrou associação da MTA, avaliada somente na posição decúbito dorsal, nos níveis axilar e xifoide com a PImáx de 0,48 e 0,46 , e com a PEmáx de 0,25 e 0,37 . No entanto, o grupo avaliado foi limitado entre 20 e 30 anos, e não avaliaram o nível umbilical, o qual apontou relação com as PImáx e PEmáx neste estudo na posição decúbito dorsal.

Os resultados do presente estudo levantaram a necessidade de padronizar uma posição para a medição da MTA, para que os resultados de avaliações clínicas e de pesquisas possam ser comparados com confiança. Sugere-se a posição decúbito dorsal pelas seguintes razões: 1) é a postura mais confortável para o participante; 2) é mais confortável para o avaliador; 3 ) a atividade do músculo diafragma é mais eficiente nessa posição ${ }^{27}$.

Como limitações do presente estudo, podemos citar a impossibilidade de determinar relações de causa e efeito e um maior risco de viés de confundimento, comuns em estudos transversais. Entretanto, comparando-se com os estudos que já investigaram a MTA nas posições ortostática e decúbito dorsal, este estudo se sobressai devido à realização da familiarização com as medidas da MTA; maior tamanho amostral e ampla faixa etária (20-79 anos). 


\section{CONCLUSÃO}

A posição tomada pelo participante para a medição da MTA interfere significativamente nos resultados a serem obtidos. Da posição ortostática para a decúbito dorsal, houve uma diminuição significativa da MTA aos níveis axilar e xifoide, e um aumento significativo ao nível umbilical. A correlação da MTA com as PImáx e PEmáx, apesar de significativa na posição decúbito dorsal, foi, no máximo, moderada. Sugere-se a adoção da posição decúbito dorsal para a medição da MTA porque, além de ser mais confortável para participante e avaliador, a atividade do músculo diafragma é mais eficiente nessa posição.

\section{AGRADECIMENTOS}

Ao Centro de Educação Física Almirante Adalberto Nunes (CEFAN), Marinha do Brasil.

\section{REFERÊNCIAS}

1. Guyton AC, Hall JE, Guyton AC. Tratado de fisiologia médica. Rio de Janeiro: Elsevier Brasil; 2006.

2. Costa D. Fisioterapia respiratória básica. São Paula: Atheneu; 1999.

3. Kakizaki F, Shibuya M, Yamazaki T, Yamada M, Suzuki H, Homma I. Preliminary report on the effects of respiratory muscle stretch gymnastics on chest wall mobility in patients with chronic obstructive pulmonary disease. Respir Care. 1999;44:409-14.

4. Rodrigues CP, Alves LA, Matsuo T, Gonçalves CG, Hayashi D. Efeito de um programa de exercícios direcionados à mobilidade torácica na DPOC. Fisioter Mov. 2012;25(2).

5. Basso RP, Regueiro EMG, Jamami M, Di Lorenzo VAP, Costa D. Relação da medida da amplitude tóraco-abdominal de adolescentes asmáticos e saudáveis com seu desempenho físico. Fisioter Mov. 2011;24(1):107-14.
6. Silva Caldeira V, Starling CCD, Britto RR, Martins JA, Sampaio RF, Parreira VF. Precisão e acurácia da cirtometria em adultos saudáveis. J Bras Pneumol. 2007;33(5):519-26.

7. Souza RB. Pressões respiratórias estáticas máximas. J Pneumol. 2002;28(3):S155-S65.

8. Society AT. ATS/ERS statement on respiratory muscle testing. Am J Respir Crit Care Med. 2002;166:518-624.

9. Gea J. La especie humana: un largo camino para el sistema respiratorio. Arch Bronconeumol. 2008;44(5):263-70.

10. Von Elm E, Altman DG, Egger M, Pocock SI, Gøtzsche PC, Vandenbroucke JP, et al. The Strengthening the Reporting of Observational Studies in Epidemiology (STROBE) statement: guidelines for reporting observational studies. Prev med. 2007;45(4):247-51.

11. Fagundes AA, Coitinho D. Vigilância alimentar e nutricional-SISVAN: orientações básicas para a coleta, processamento, análise de dados e informação em serviços de saúde. Série A Normas e manuais técnicos: Ministério da Saúde; 2004.

12. Marfell-Jones MJ, Stewart AD, de Ridder J. International standards for anthropometric assessment. Wellington, New Zealand: International Society for the Advancement of Kinanthropometry; 2012 .

13. Matsudo S, Araújo T, Marsudo V, Andrade D, Andrade E, Braggion G. Questinário internacional de atividade f1sica (IPAQ): estudo de validade e reprodutibilidade no Brasil. Rev bras ativ fís saúde. 2001;6(2):05-18.

14. Pedrini A, Gonçalves MA, Leal BE, Santos Yamaguti WP, Paulin E. Comparação entre as medidas de cirtometria tóraco-abdominal realizadas em decúbito dorsal e em ortostatismo. Fisiot e Pesq. 2013;20(4):373-8.

15. Fattini CA, Dangelo JG. Anatomia Humana Sistêmica e Segmentar. 3. ed. São Paulo: Atheneu; 2007.

16. Lanza FD, Camargo AA, Archija LR, Selman JP, Malaguti C, Dal Corso $\mathrm{S}$. Chest wall mobility is related to respiratory muscle strength and lung 
volumes in healthy subjects. Respir Care. 2013;58(12):2107-12.

17.Sigmound R. Estatística nãoparamétrica. São Paulo: McGraw-20 Hill; 2004.

18. Hopkins WG, Marshall SW, Batterham AM, Hanin J. Progressive statistics for studies in sports medicine and exercise science. Med Sci Sports Exerc. 2009;41:3-12.

19. Silva AB, Mendes RG, Silva ES, Picchi PC, Di Lorenzo VAP, Paulucci HL. Medida da amplitude tóraco-abdominal como método de avaliação dos movimentos do tórax e abdome em indivíduos jovens saudáveis. Fisioter Bras. 2006;7(1):25-9.

20. Costa D, Forti E, Barbalho-Mou$\lim$ M, Rasera-Junior I. Estudo dos volumes pulmonares e da mobilidade toracoabdominal de portadoras de obesidade mórbida, submetidas à cirurgia bariátrica, tratadas com duas diferentes técnicas de fisioterapia. Rev Bras Fisioter. 2009;13(4).

21. Basso RP, Regueiro EMG, Jamami M, Di Lorenzo VAP, Costa D. Relação da medida da amplitude tóraco-abdominal de adolescentes asmáticos e saudáveis com seu desempenho físico. Fisioter Mov. 2017;24(1).

22. Jamami M, Pires VA, Oirshi J, Costa D. Efeitos da intervenção fisioterápica na reabilitação pulmonar de pacientes com doença pulmonar obstrutiva crônica (DPOC). Fisioter Pesq. 1999;6(2):140-53
23. Malaguti C, Rondelli RR, Souza LM, Domingues M, Dal Corso S. Reliability of chest wall mobility and its correlation with pulmonary function in patients with chronic obstructive pulmonary disease. Respir Care. 2009;54(12):1703-11.

24. Druz WS, Sharp JT. Activity of respiratory muscles in upright and recumbent humans. J Appl Physiol. 1981;51(6):1552-61.

25. Clarysse I, Demedts M. Human esophageal pressures and chest wall configuration in upright and head-down posture. J Appl Physiol. 1985;59(2):401-7.

26. Sackner MA, Gonzalez H, Rodriguez M, Belsito A, Sackner DR, Grenvik S. Assessment of Asynchronous and Paradoxic Motion between Rib Cage and Abdomen in Normal Subjects and in Patients with Chronic Obstructive Pulmonary Disease. Am Rev Respir Dis. 1984;130(4):588-93.

27. Verschakelen JA, Demedts MG. Normal thoracoabdominal motions. Influence of sex, age, posture, and breath size. Am J Respir Crit Care Med. 1995;151(2):399-405. 